ARTICLE

Received 27 Nov 2012 | Accepted 20 Mar 2013 | Published 23 Apr 2013 DOI: 10.1038/ncomms2771

\title{
Solid-state electronic spin coherence time approaching one second
}

\author{
N. Bar-Gill1,2, L.M. Pham³ ${ }^{3}$ A. Jarmola4 ${ }^{4}$ D. Budker ${ }^{4,5} \&$ R.L. Walsworth ${ }^{1,2}$
}

Solid-state spin systems such as nitrogen-vacancy colour centres in diamond are promising for applications of quantum information, sensing and metrology. However, a key challenge for such solid-state systems is to realize a spin coherence time that is much longer than the time for quantum spin manipulation protocols. Here we demonstrate an improvement of more than two orders of magnitude in the spin coherence time $\left(T_{2}\right)$ of nitrogen-vacancy centres compared with previous measurements: $T_{2} \approx 0.6 \mathrm{~s}$ at $77 \mathrm{~K}$. We employed dynamical decoupling pulse sequences to suppress nitrogen-vacancy spin decoherence, and found that $T_{2}$ is limited to approximately half of the longitudinal spin relaxation time over a wide range of temperatures, which we attribute to phonon-induced decoherence. Our results apply to ensembles of nitrogen-vacancy spins, and thus could advance quantum sensing, enable squeezing and many-body entanglement, and open a path to simulating driven, interactiondominated quantum many-body Hamiltonians.

\footnotetext{
${ }^{1}$ Harvard-Smithsonian Center for Astrophysics, Cambridge, Massachusetts 02138, USA. ${ }^{2}$ Department of Physics, Harvard University, Cambridge, Massachusetts 02138, USA. ${ }^{3}$ School of Engineering and Applied Sciences, Harvard University, Cambridge, Massachusetts 02138, USA. ${ }^{4}$ Department of Physics, University of California, Berkeley, California 94720-7300, USA. ${ }^{5}$ Nuclear Science Division, Lawrence Berkeley National Laboratory, Berkeley, California 94720, USA. Correspondence and requests for materials should be addressed to N.B.-G. (email: nbar-gill@cfa.harvard.edu) or to R.L.W. (email: rwalsworth@cfa.harvard.edu).
} 
$\mathrm{n}$ recent years, the electronic spin of the negatively charged nitrogen-vacancy (NV) colour centre in diamond has become a leading platform for applications ranging from quantum information processing ${ }^{1}$ to quantum sensing and metrology ${ }^{2-4}$. Importantly, the NV spin-state can be optically initialized and detected on a timescale of $\sim 300$ ns (ref. 5) and coherently driven at up to gigahertz rates ${ }^{5}$. Single NV centres in isotopically engineered high-purity diamond can possess long electronic spin coherence times on the order of a few millisecond at room temperature ${ }^{6}$. By applying dynamical decoupling sequences, similar coherence times can be achieved for single $\mathrm{NV}$ centres $^{7-9}$ and $\mathrm{NV}$ ensembles ${ }^{10}$ in diamond containing higher impurity concentration.

Here we apply dynamical decoupling techniques to ensembles of NV centres over a range of temperatures $(77 \mathrm{~K}-300 \mathrm{~K})$ in order to suppress both decoherence ${ }^{10}$ and phononic spin relaxation $^{11}$. We demonstrate an extension of the $\mathrm{NV}$ spin coherence time $\left(T_{2}\right)$ to $T_{2} \approx 0.6 \mathrm{~s}$ at $77 \mathrm{~K}$, which corresponds to an improvement of more than two orders of magnitude compared with previous measurements ${ }^{6,10}$ and is on par with the longest coherence times achieved for electronic spins in any solid-state system ${ }^{12}$. Over a wide range of temperatures we also find that the NV spin $T_{2}$ is limited to approximately half of the longitudinal spin relaxation time $\left(T_{1}\right), T_{2} \approx 0.5 T_{1}$, a finding that could be relevant to other solid-state spin defects (such as $\mathrm{P}$ donors in $\mathrm{Si}$ ). The present result of $\mathrm{NV} T_{2}$ approaching $1 \mathrm{~s}$, in combination with single $\mathrm{NV}$ optical addressability and the practicality and scalability of diamond, advance NV centres to the forefront of candidates for quantum information, simulation and sensing applications ${ }^{1,2}$.

\section{Results}

Coherence decay and longitudinal relaxation. As an example of our data, we plot in Fig. 1 the measured NV spin coherence as a function of time for Carr-Purcell-Meiboom-Gill (CPMG) pulse sequences $^{13}$ with different numbers of pulses $n$, at room temperature $T=300 \mathrm{~K}$ (a) and at $T=160 \mathrm{~K}$ (b), for an isotopically pure $\left(0.01 \%{ }^{13} \mathrm{C}\right)$ diamond sample with nitrogen density $\sim 10^{15} \mathrm{~cm}^{-3}$ and NV density $\sim 3 \times 10^{12} \mathrm{~cm}^{-3}$ (see Methods section for details). For each of these decoherence curves, we extracted $T_{2}$ by fitting the data to a stretched exponential function $f(t)=\exp \left[-\left(t / T_{2}\right)^{p}\right]$, where the $p$ parameter is related to the dynamics of the spin environment and inhomogeneous broadening due to ensemble averaging ${ }^{14}$. For both temperatures $T_{2}$ increases with the number of pulses in the CPMG sequence and is limited to about half of $T_{1}$ : at $300 \mathrm{~K}$, $T_{2}=3.3(4) \mathrm{ms}$ and $T_{1}=6.0(4) \mathrm{ms}$; and at $160 \mathrm{~K}, T_{2}=40(8) \mathrm{ms}$ and $T_{1}=77(5) \mathrm{ms}$.

Coherence versus number of pulses at different temperatures. Figure 2a summarizes the measured dependence of $T_{2}$ on the number of CPMG pulses $n$ for several temperatures ranging from liquid-nitrogen temperature $(77 \mathrm{~K})$ to room temperature $(300 \mathrm{~K})$. Notably, at liquid nitrogen temperature, an 8192-pulse CPMG sequence achieved a coherence time of $T_{2}=580 \mathrm{~ms}$, which is more than two orders of magnitude longer than previous NV $T_{2}$ measurements ${ }^{6,10}$. Accumulated pulse errors limited sequences with larger $n$. At $T=160 \mathrm{~K}$, a temperature that can be reached using thermoelectric cooling rather than cryogenic fluids, we found $T_{2}=40(8) \mathrm{ms}$, which is an order of magnitude longer than any previous $\mathrm{NV} T_{2}$ measurement. Thus, combined dynamical decoupling and thermoelectric cooling provides a practical way to greatly increase the NV spin coherence time, which could benefit many applications of $\mathrm{NV}$ ensembles, for example, precision magnetometry ${ }^{10}$ and rotation sensing ${ }^{15,16}$.
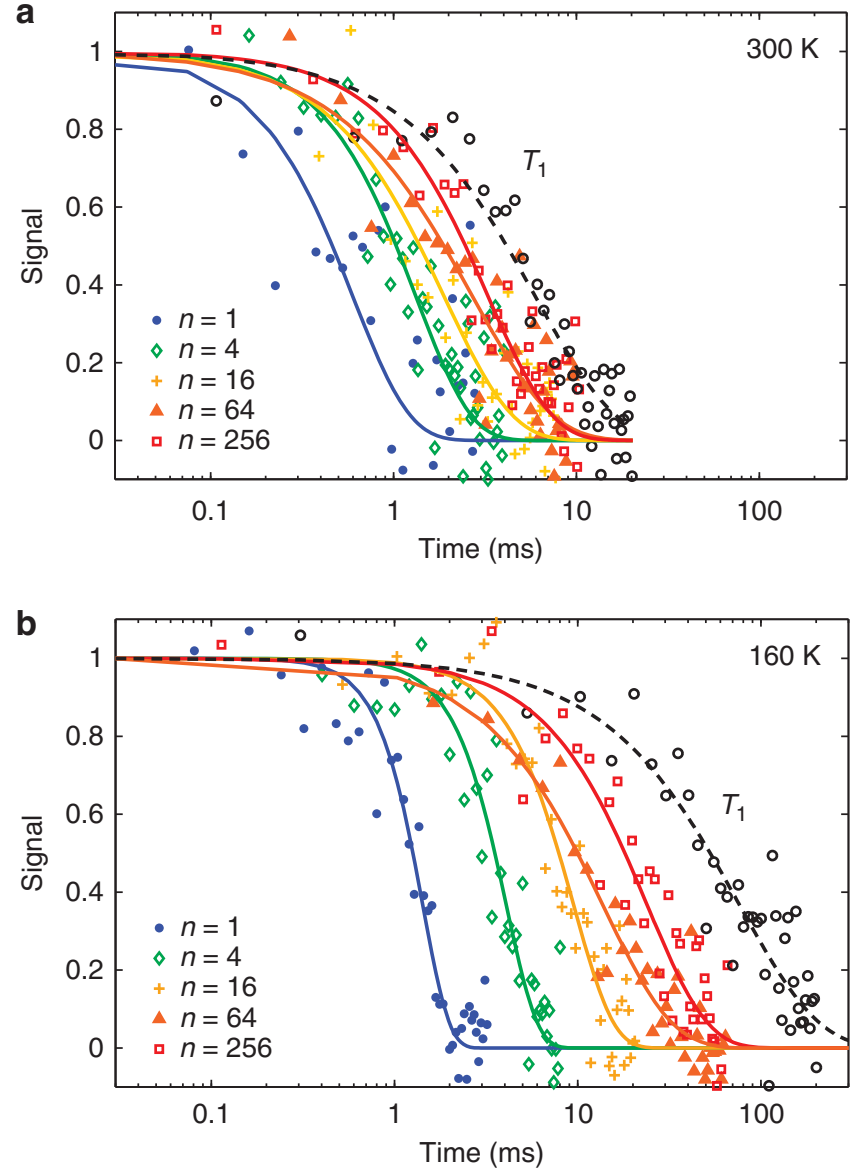

Figure 1 | Measured coherence decay and longitudinal relaxation. Measured NV spin coherence decay used to determine $T_{2}$ for $n$-pulse CPMG sequences (coloured data points and solid line fits to data), and longitudinal spin relaxation used to determine $T_{1}$ (black data points and dashed line fits to data), at (a) $T=300 \mathrm{~K}$ and (b) $T=160 \mathrm{~K}$.

The CPMG pulse sequence used here is quite robust against noise (for example, external magnetic field fluctuations) and pulse errors, allowing us to apply thousands of pulses. This robustness allows us to extract a near-ideal limit to the coherence time $T_{2}$ achievable with perfect pulses, although it is obtained only for one specific spin component. The other transverse spin component is more sensitive to pulse errors and has a shorter decay time, which can be improved to $T_{2}$ with more optimal pulses and symmetrized pulse sequences (such as XY). The longitudinal spin component is unaffected by these pulse errors and decays with a timescale given by $T_{1}$. We note that the measurement scheme that we use (see Methods) allows for common-mode rejection of noise, but does not remove the effect of pulse errors. We noticed a modest reduction in signal contrast and increased noise for pulse sequences of $\sim 10,000$ pulses or more (Fig. 3). The resulting reduction in signal-to-noise, together with the long integration times needed, currently prevents us from increasing the coherence time to beyond $\approx 0.6 \mathrm{~s}$ (see Methods for additional technical details). In future work, we will address these issues using various approaches, including more robust composite pulses ${ }^{17}$ that could be better suited to manipulate an inhomogeneously broadened ensemble. This will allow the long coherence times demonstrated here to be employed for enhanced spin sensing and quantum information processing. 

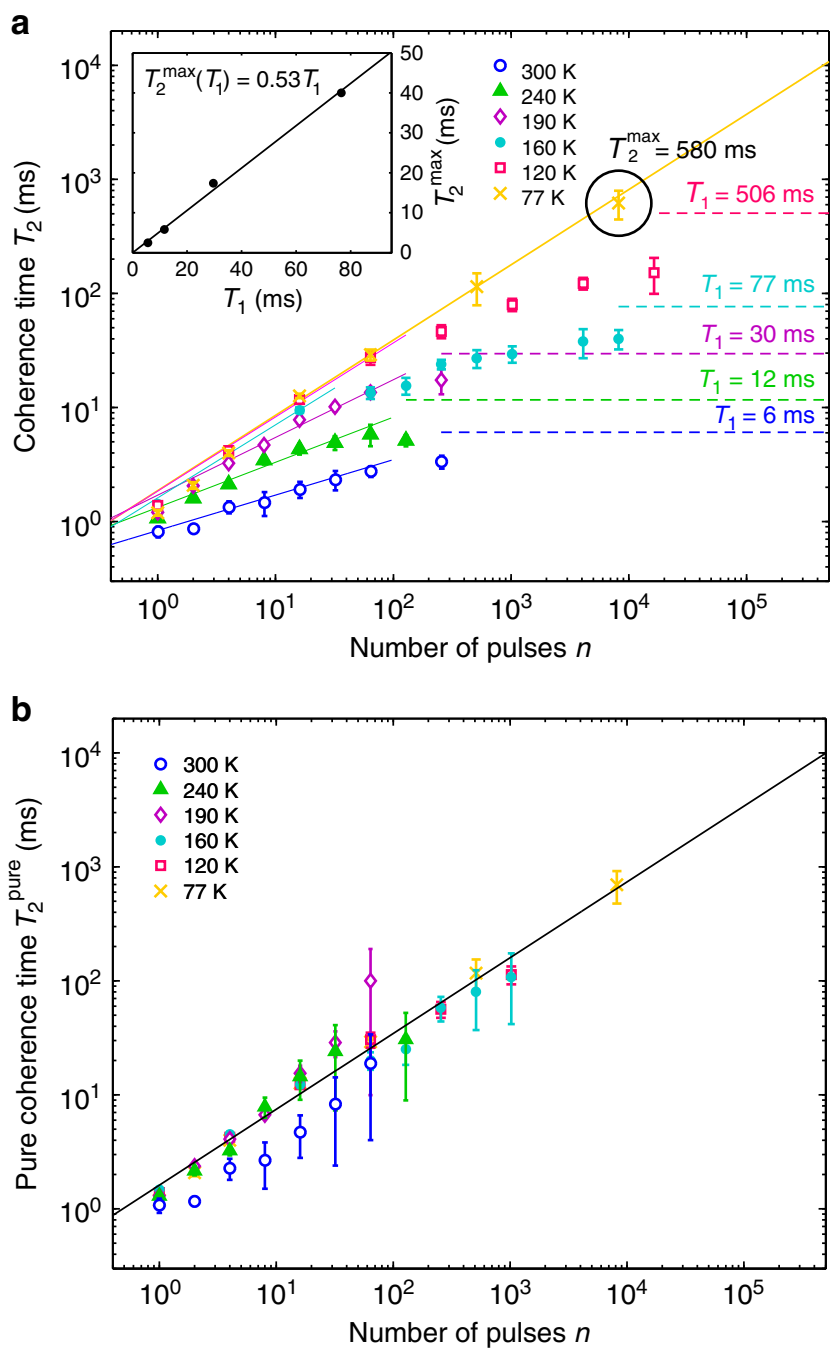

Figure 2 | Measured coherence time and extracted 'pure' coherence time for different numbers of pulses at different temperatures.

(a) Measured coherence time $T_{2}$ as a function of the number of pulses $n$ in the applied CPMG sequence, for temperatures from 77 to $300 \mathrm{~K}$. Error bars indicate $1 \sigma$ uncertainty in the fit used to extract $T_{2}$ (using decoherence curves as shown in Fig. 1). For $T=160 \mathrm{~K}$ and above, the corresponding measured longitudinal relaxation time $T_{1}$ is plotted as a dashed line. The black circle indicates the longest $T_{2}=580(210) \mathrm{ms}$ for $n=8,192$ at $77 \mathrm{~K}$. Inset: scaling of the maximum coherence time as a function of $T_{1}: T_{2}^{\max }=0.53(2) T_{1}$. (b) NV coherence time with the phononic relaxation rate $\left[0.53(2) T_{1}\right]^{-1}$ subtracted to extract the 'pure' spin-environment-induced decoherence, with scaling behaviour of $T_{2}^{\text {pure }} \propto n^{0.67(3)}$ (see text).

Relationship between maximal coherence and relaxation times. The $T_{1}$ relaxation time measured for each temperature is also indicated in Fig. 2a (except for $77 \mathrm{~K}$, which had $T_{1}>10 \mathrm{~s}$ ). In the figure inset, we plot the maximum $T_{2}$ achieved vs. $T_{1}$ at each temperature in the interval of $160-300 \mathrm{~K}$ and find $T_{2}^{\max }=0.53(2) T_{1}$, which differs starkly from the previously expected $T_{2}$ limit of $2 T_{1}$ (ref. 9). Under the assumption that spin-phonon coupling only causes spin-lattice relaxation $\left(T_{1}\right)$, one cannot recover the measured $T_{2} \approx 0.5 T_{1}$ limit, even taking into account the possibility of unequal relaxation rates between the three ground-state NV spin sublevels. This can be seen by assuming that only state-changing relaxation

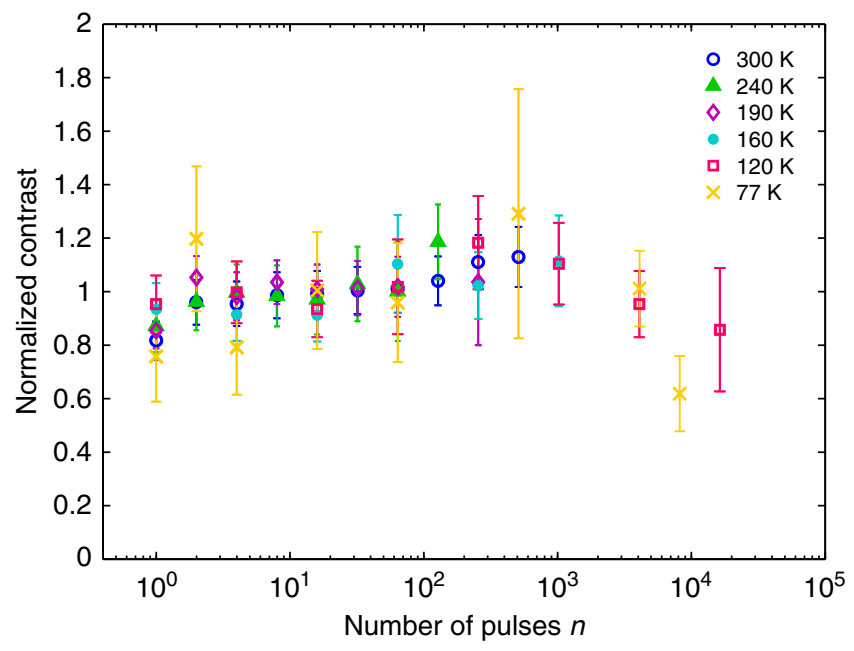

Figure 3 | Measured normalized signal contrast $A(T, n)$ as a function of the number of CPMG pulses $\boldsymbol{n}$ for different temperatures $\boldsymbol{T}$. For each temperature, the contrast of the measured signal $m(t)$ for a given pulse sequence CPMG- $n$ (see Methods), denoted $A_{n}^{T}$, is extracted from the fit to a stretched exponential (see Supplementary Methods). It is then normalized against the average contrast of all pulse sequences at that temperature, denoted $\left\langle A_{n}^{T}\right\rangle_{n}$. Therefore, the plotted normalized contrast is given by $A(T, n)=A_{n}^{T} /\left\langle A_{n}^{T}\right\rangle_{n}$.

processes exist and, therefore, the decoherence rate between two states $|a\rangle$ and $|b\rangle, \gamma_{2}^{a b}$, is given by ${ }^{18}$

$$
\gamma_{2}^{a b}=\frac{1}{2}\left(\sum_{n \neq a} \gamma_{1}^{a \rightarrow n}+\sum_{n \neq b} \gamma_{1}^{b \rightarrow n}\right),
$$

where $\gamma_{1}^{m \rightarrow n}$ is the transition rate from state $|m\rangle$ to state $|n\rangle$. Solving rate equations allows one to calculate the measured relaxation rate $\left(\gamma_{1}=1 / T_{1}\right)$ as a function of these transition rates, and thus compare it with the decoherence rate $\left(\gamma_{2}=1 / T_{2}\right)$ given by Equation (1). Through this approach one can show that for a two-level system, at temperatures ranging from zero to infinite temperature, the relation is always $T_{2}=2 T_{1}$. For three or more levels the coefficient changes, but the general statement $T_{2}>T_{1}$ holds (see Supplementary Methods for further details). Therefore we conclude that in our case, as we measure $T_{2}<T_{1}$, spinphonon coupling contributes directly and significantly to NV spin decoherence ${ }^{19}$. Such phonon-induced decoherence is generally relevant to any quantum system in which transitions between two levels can be driven by a two-phonon (Raman) process ${ }^{11}$, and could have a role in the coherence properties of many other solidstate defects. At lower temperatures, to be studied in future work, spin-spin interactions should dominate both $T_{1}$ and $T_{2}$, and thus we expect $T_{2}^{\max }$ will deviate from the $\approx 0.5 T_{1}$ limit demonstrated here (see Supplementary Methods).

\section{Discussion}

It is evident from Fig. 2a that the scaling of the coherence time with the number of CPMG pulses varies with temperature. In order to study the scaling behaviour of the 'pure' spinenvironment-induced decoherence, we subtracted from the measured decoherence rate the temperature dependent phononic rate $1 /\left[0.53(2) T_{1}\right]$ (see inset of Fig. $2 a$ ). The corrected coherence time $T_{2}^{\text {pure }}$ is plotted against the number of CPMG pulses $n$ in Fig. 2b, exhibiting striking universal behaviour for all temperatures. We fit the corrected data to a power law scaling, and 
a

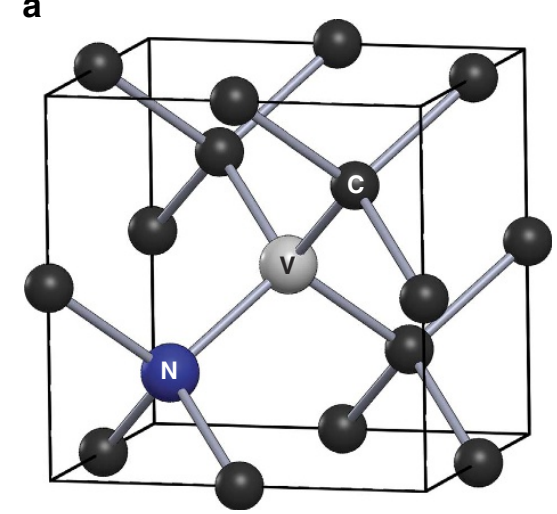

b

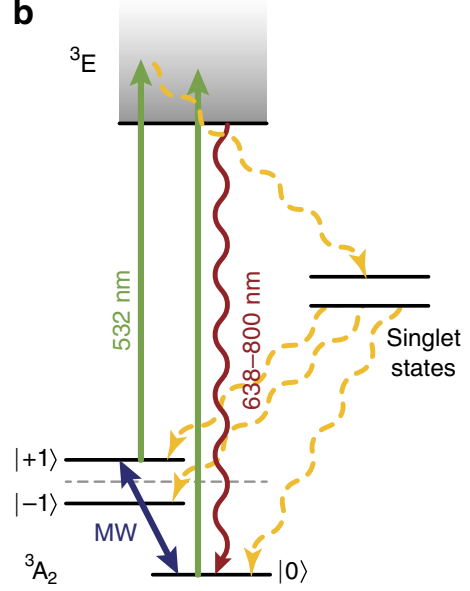

$\mathbf{C}$

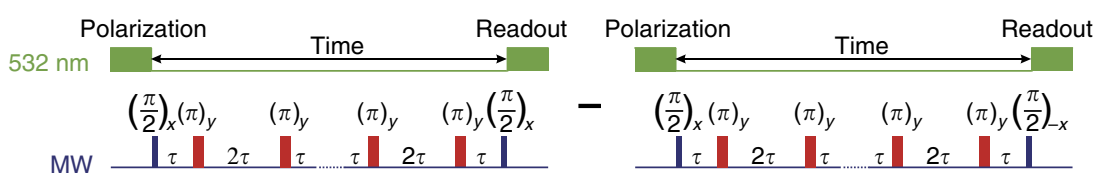

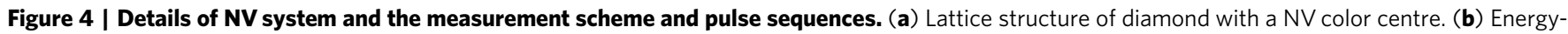
level schematic of the negatively charged NV centre. (c) Measurement scheme: initialization and readout using $532 \mathrm{~nm}$ light, between which CPMG dynamical decoupling MW control sequences are applied. The sequence is repeated with the phase of the last $\pi / 2$ pulse flipped (from $x$ to $-x$ ) for normalization (see text).

find $T_{2}^{\text {pure }} \propto n^{0.67(3)}$. This value is consistent with the expected scaling power of $2 / 3$ for a Lorentzian noise spectrum of an electronic spin bath ${ }^{14,20}$.

Even though our measurements were performed on an isotopically pure sample $\left(0.01 \%{ }^{13} \mathrm{C}\right)$, we expect that similar results can be obtained for natural abundance diamond $(1.1 \%$ ${ }^{13} \mathrm{C}$ ), as the dynamical decoupling sequences we employ are also effective in suppressing dephasing caused by the nuclear spin bath $^{8,10}$. However, special care must be taken in aligning the applied static magnetic field along the NV axis for natural abundance diamond since for ensembles of NVs in the presence of ${ }^{13} \mathrm{C}$ nuclear spins, additional decoherence is caused by variations in the effective Larmor frequency of nearby nuclear spins due to magnetic field misalignment ${ }^{21,22}$. At present we have performed measurements on one additional sample obtaining consistent results (see Methods). From this reproducibility and the ensemble nature of our experiments, we conclude that the data presents the behaviour of typical NVs, averaged over inhomogeneous broadening effects such as strain.

The demonstrated improvement in coherence times for large ensembles of NV spins is directly relevant to enhanced metrology and magnetic field sensitivity ${ }^{23,24}$, which scales as $\left(N_{\mathrm{NV}} T_{2}\right)^{-1 / 2}$, where $N_{\mathrm{NV}}$ is the number of sensing $\mathrm{NVs}^{2,10,25}$. Thus, the hundred-fold increase in $T_{2}$ measured in this work at $77 \mathrm{~K}$ enables a 10-fold improvement in magnetic field sensitivity. Using samples with higher densities of NVs could further improve this sensitivity; although at high NV concentrations ( $\gtrsim 1$ p.p.b.) the coherence time may be limited by NV-NV interactions, as CPMG pulse sequences affect the NV spin and its $\mathrm{NV}$ spin-bath at the same time, thus cancelling the decoupling effect. Other techniques, such as WAHUHA and MREV pulse sequences $^{26,27}$, may be applied to address this issue.

We note that achieving long NV spin coherence times in diamond samples with high impurity concentration ( $\sim 1$ p.p.m.) is a crucial step towards creating non-classical states of NV ensembles. Such non-classical states could form the basis for high-sensitivity quantum metrology, potentially allowing significantly improved sensitivity and bandwidth ${ }^{28,29}$, and could also serve as a resource for quantum information protocols. To observe significant entanglement between neighbouring NV centres, their decoherence rate must be small compared with the frequency associated with their interaction. For a realistic diamond sample with $[\mathrm{N}] \sim 1$ p.p.m. and [NV] $\sim 10$ p.p.b., coherence times larger than $\sim 50 \mathrm{~ms}$ are needed for significant entanglement, which is within reach given the results presented here. For example, collective spin squeezing using $L_{Z}^{2}$ one-axis squeezing techniques ${ }^{30}$ could be created through application of pulse sequences that average-out the $X, Y$ components of the spin-spin dipolar coupling ${ }^{31}$. Such pulse sequences can be straightforwardly applied in conjunction with the CPMG pulse sequences used here for extending the NV spin coherence time.

In conclusion, we demonstrated more than two orders of magnitude improvement in the coherence time $\left(T_{2}\right)$ of ensembles of NV electronic spins in diamond compared with previous results: up to $T_{2} \approx 0.6 \mathrm{~s}$ by combining dynamical decoupling control sequences with cryogenic cooling to $77 \mathrm{~K}$; and $T_{2} \simeq 40 \mathrm{~ms}$ for temperatures achievable via thermoelectric cooling ( $>160 \mathrm{~K})$. By studying the dependence of $T_{2}$ and of the $\mathrm{NV}$ spin relaxation time $\left(T_{1}\right)$ on temperature, we identified an effective limit of $T_{2} \approx 0.5 T_{1}$, which we attribute to phonon-induced decoherence. Given this limit, we expect that for low NV densities an electronic spin $T_{2}$ of a few seconds should be achievable at liquid-nitrogen temperatures $(T=77 \mathrm{~K})$.

The greatly extended NV spin coherence time presented in this work, which does not require an optimally chosen NV centre, could form the building block for wide-ranging applications in quantum information, sensing, and metrology in the solidstate $^{32,33}$. In particular, the fact that such long coherence times can be achieved with high-density ensembles of NVs suggests that spin squeezing and highly entangled states can be created, since $T_{2}>\mathrm{NV}-\mathrm{NV}$ dipolar interaction time. Finally, this work could 
provide a key step toward realizing interaction-dominated topological quantum phases in the solid-state, as well as a large family of driven many-body quantum Hamiltonians ${ }^{34}$.

\section{Methods \\ NV structure. The NV centre consists of a substitutional nitrogen atom} and a vacancy occupying adjacent lattice sites in the diamond crystal (Fig. 4a). The electronic ground state is a spin triplet (Fig. $4 \mathrm{~b}$ ), in which the $m_{\mathrm{s}}=0$ and \pm 1 sublevels experience a $\sim 2.87 \mathrm{GHz}$ zero-field splitting. The $\mathrm{NV}$ centre can be rendered an effective two-level system by applying a static magnetic field to further split the $m_{\mathrm{s}}= \pm 1$ states and addressing, for example, the $m_{\mathrm{s}}=0$ and +1 Zeeman sublevels. The NV spin can be initialized with optical excitation, detected via the state-dependent fluorescence intensity, and coherently manipulated using microwave pulse sequences ${ }^{35}$.

Experimental set-up. We performed measurements on an isotopically pure $\left(0.01 \%{ }^{13} \mathrm{C}\right)$ diamond sample (Element Six) with nitrogen density $\sim 10^{15} \mathrm{~cm}^{-3}$ and NV density $\sim 3 \times 10^{12} \mathrm{~cm}^{-3}$. The sample was mounted inside a continuousflow cryostat (Janis ST-500) with active temperature control (Lakeshore 331). Optical excitation was done with a 532-nm laser beam focused on the diamond surface through a microscope objective $(\mathrm{NA}=0.6)$, and the resulting NV fluorescence was collected through the same objective and directed to a multimode fibre coupled to a single-photon counting module (Perkin-Elmer). The $\sim 30 \mu^{3}$ detection volume contained $\sim 100 \mathrm{NV}$ centres. Microwave control pulses were delivered to the NV spins using a 70- $\mu \mathrm{m}$ diameter copper wire at the diamond surface. The experiments were performed in an unshielded environment.

We measured an additional, higher density isotopically pure $\left(0.01 \%{ }^{13} \mathrm{C}\right)$ diamond sample (Element Six), with a nitrogen density $\sim 10^{17} \mathrm{~cm}^{-3}$ and NV density $\sim 10^{15} \mathrm{~cm}^{-3}$. Owing to the higher density of the sample, the coherence times achieved were consistently lower. At $T=77 \mathrm{~K}$ with $n=4,096$ pulses, we obtained a coherence time of $T_{2}=50(9) \mathrm{ms}$, with a scaling of the coherence time with the number of pulses of $T_{2}(n)=n^{0.54(1)}$.

Pulse sequences. We applied multi-pulse control sequences to decouple NV spins from the magnetic environment and thus extend the coherence time. Specifically, we used the CPMG pulse sequence ${ }^{13}$ (Fig. 4c), with a varying number $n$ of $\pi$ control pulses. It has been shown previously ${ }^{7-10}$ that such pulse sequences are effective in extending the coherence time $T_{2}$ of NV spins. The coherence time increases as a power law of the number of pulses $T_{2} \propto n^{s}$, where the specific scaling $s$ is determined mainly by the spin bath surrounding the $\mathrm{NV}^{14}$ until spin-lattice relaxation begins limiting $T_{2}$ (assuming no pulse-error effects).

Measurement normalization schemes. The coherence measurements were performed by applying the CPMG pulse sequence with the last $\pi / 2$ pulse either along the $x$ axis or the $-x$ axis (Fig. 4c). The two signals, labelled $r_{1}$ and $r_{2}$, were then subtracted and normalized to give the measurement results $m(t)=\left(r_{1}-r_{2}\right) /$ $\left(r_{1}+r_{2}\right)$ such that common-mode noise is rejected. The final signal $m(t)$ is proportional to the coherence of the $\mathrm{NV}$ spin, defined as the magnitude of the off-diagonal density matrix element of the $m_{\mathrm{s}}=0,+1$ two-level system $C(t)=\operatorname{Trace}\left[\rho(t) S_{x}\right]$ (with $\rho(t)$ being the density matrix, and $S_{x}$ the transverse spin operator), at the end of the pulse sequence (before the final $\pi / 2$ pulse). For a given pulse sequence (with $n$ pulses), the NV spin coherence as a function of time was measured by varying the free precession time between pulses and thus the total sequence time.

Measurements of the NV longitudinal spin relaxation followed a procedure similar to that used for the coherence measurements, with one of the two 'common-mode' measurements initialized to $m_{\mathrm{s}}=0$ (using an optical pumping pulse) and the other initialized to $m_{\mathrm{s}}=+1$ (using an optical pumping pulse followed by a MW $\pi$ pulse). The normalized signal is proportional to the population difference between the spin states at time $t$, allowing measurement of the longitudinal spin relaxation and fitting to extract the $T_{1}$ relaxation time.

The normalized signal contrast (Fig. 3 ) is calculated by extracting the contrast of each decoherence curve (for example, Fig. 1) through a fit to a decaying stretched exponential (see Supplementary Methods), and then normalizing this contrast by the average contrast for all pulse sequences (at a given temperature).

Pulse errors. The effect of pulse errors is due to amplitude and timing jitter originating in our microwave source and pulse generator. One aspect of the timing jitter is related to the pulse generator timing, which includes an output jitter of $\sim 2$ ns (negligible compared with the long duration of the pulse sequences) and a timebase accuracy of $\sim 10^{-4}$. The other aspect of the timing jitter is related to the timebase of the microwave synthesizer, which has an accuracy of $\sim 10^{-10}$. Timing jitter for both the pulser and the microwave synthesizer can be improved by using an external timebase.

\section{References}

1. Ladd, T. et al. Quantum computers. Nature 464, 45-53 (2010).

2. Taylor, J. M. et al. High-sensitivity diamond magnetometer with nanoscale resolution. Nat. Phys. 4, 810-816 (2008).

3. Pham, L. M. et al. Magnetic field imaging with nitrogen-vacancy ensembles. New J. Phys. 13, 045021 (2011).

4. Dolde, F., Fedder, H., Doherty, M. \& Nbauer, T. Electric-field sensing using single diamond spins. Nat. Phys. 7, 459-463 (2011).

5. Fuchs, G. D., Dobrovitski, V. V., Toyli, D. M., Heremans, F. J. \& Awschalom, D. D. Gigahertz dynamics of a strongly driven single quantum spin. Science 326, 1520-1522 (2009).

6. Balasubramanian, G. et al. Ultralong spin coherence time in isotopically engineered diamond. Nat. Mater. 8, 383-387 (2009).

7. de Lange, G., Wang, Z. H., Rist, D., Dobrovitski, V. V. \& Hanson, R. Universal dynamical decoupling of a single solid-state spin from a spin bath. Science 330, 60-63 (2010).

8. Ryan, C. A., Hodges, J. S. \& Cory, D. G. Robust decoupling techniques to extend quantum coherence in diamond. Phys. Rev. Lett. 105, 200402 (2010).

9. Naydenov, B. et al. Dynamical decoupling of a single-electron spin at room temperature. Phys. Rev. B 83, 081201 (2011).

10. Pham, L. M. et al. Enhanced solid-state multispin metrology using dynamical decoupling. Phys. Rev. B 86, 045214 (2012).

11. Jarmola, A., Acosta, V. M., Jensen, K., Chemerisov, S. \& Budker, D. Temperature- and magnetic-field-dependent longitudinal spin relaxation in nitrogen-vacancy ensembles in diamond. Phys. Rev. Lett. 108, 197601 (2012).

12. Tyryshkin, A. et al. Electron spin coherence exceeding seconds in high-purity silicon. Nat. Mater. 11, 143-147 (2012).

13. Meiboom, S. \& Gill, D. Modified spin-echo method for measuring nuclear relaxation times. Rev. Sci. Instrum. 29, 688-691 (1958).

14. Bar-Gill, N. et al. Suppression of spin-bath dynamics for improved coherence of multi-spin-qubit systems. Nat. Commun. 3, 858 (2012).

15. Ledbetter, M. P., Jensen, K., Fischer, R., Jarmola, A. \& Budker, D. Gyroscopes based on nitrogen-vacancy centers in diamond. Phys. Rev. A 86, 052116 (2012).

16. Ajoy, A. \& Cappellaro, P. Stable three-axis nuclear-spin gyroscope in diamond. Phys. Rev. A 86, 062104 (2012).

17. Shim, J. H., Niemeyer, I., Zhang, J. \& Suter, D. Robust dynamical decoupling for arbitrary quantum states of a single NV center in diamond. Europhys. Lett. 99, 40004 (2012).

18. Cohen-Tannoudji, C., Dupont-Roc, J. \& Grynberg, G. Atom-Photon Interactions: Basic Processes and Applications (John Wiley \& Sons, 1998).

19. Doherty, M. W. et al. Theory of the ground-state spin of the $\mathrm{NV}^{-}$center in diamond. Phys. Rev. B 85, 205203 (2012).

20. de Sousa, R. in Electron Spin Resonance and Related Phenomena in Low-Dimensional Structures Vol. 115 (ed. Fanciulli, M.) 183-220 (Springer 2009).

21. Stanwix, P. L. et al. Coherence of nitrogen-vacancy electronic spin ensembles in diamond. Phys. Rev. B 82, 201201 Springer, (2010).

22. Maze, J. R., Taylor, J. M. \& Lukin, M. D. Electron spin decoherence of single nitrogen-vacancy defects in diamond. Phys. Rev. B 78, 094303 (2008).

23. Pham, L. M. et al. Enhanced metrology using preferential orientation of nitrogen-vacancy centers in diamond. Phys. Rev. B 86, 121202 (2012).

24. Le Sage, D. et al. Efficient photon detection from color centers in a diamond optical waveguide. Phys. Rev. B 85, 121202 (2012).

25. Acosta, V. M. et al. Diamonds with a high density of nitrogen-vacancy centers for magnetometry applications. Phys. Rev. B 80, 115202 (2009).

26. Waugh, J. S., Huber, L. M. \& Haeberlen, U. Approach to high-resolution NMR in solids. Phys. Rev. Lett. 20, 180-182 (1968).

27. Rhim, W.-K., Elleman, D. D. \& Vaughan, R. W. Analysis of multiple pulse NMR in solids. J. Chem. Phys. 59, 3740-3749 (1973).

28. Gross, C., Zibold, T., Nicklas, E., Estve, J. \& Oberthaler, M. Nonlinear atom interferometer surpasses classical precision limit. Nature 464, 1165-1169 (2010).

29. Gross, C. et al. Atomic homodyne detection of continuous-variable entangled twin-atom states. Nature 480, 219-223 (2011).

30. Kitagawa, M. \& Ueda, M. Squeezed spin states. Phys. Rev. A 47, 5138-5143 (1993).

31. Cappellaro, P. \& Lukin, M. D. Quantum correlation in disordered spin systems: applications to magnetic sensing. Phys. Rev. A 80, 032311 (2009).

32. van der Sar, T. et al. Decoherence-protected quantum gates for a hybrid solidstate spin register. Nature 484, 82-86 (2012).

33. Maze, J. R. et al. Nanoscale magnetic sensing with an individual electronic spin in diamond. Nature 455, 644-647 (2008)

34. Yao, N. Y. et al. Topological flat bands from dipolar spin systems. Phys. Rev. Lett. 109, 266804 (2012).

35. Childress, L. et al. Coherent dynamics of coupled electron and nuclear spin qubits in diamond. Science 314, 281-285 (2006). 


\section{Acknowledgements}

This research has been supported by the DARPA QuASAR, QuEST and MURI-QuISM programs, NSF, IMOD, and the NATO Science for Peace Programme. We gratefully acknowledge the provision of diamond samples by Element Six, and helpful technical discussions with Ran Fischer, Daniel Twitchen, Matthew Markham, Alastair Stacey, Keigo Arai, Chinmay Belthangady, David Glenn and David Le Sage.

\section{Author contributions}

All authors discussed the results, analysed the data and commented on the manuscript.

\section{Additional information}

Supplementary Information accompanies this paper at http://www.nature.com/ naturecommunications

Competing financial interests: The authors declare no competing financial interests.

Reprints and permission information is available online at http://npg.nature.com/ reprintsandpermissions/

How to cite this article: Bar-Gill, N. et al. Solid-state electronic spin coherence time approaching one second. Nat. Commun. 4:1743 doi: 10.1038/ncomms2771 (2013). 